\title{
THREE-LEGGED WIRELESS MINIATURE ROBOTS FOR MASS-SCALE OPERATIONS AT THE SUB-ATOMIC SCALE
}

\author{
Sylvain Martel, Mark Sherwood, Chad Helm, William Garcia de Quevedo. Timothy Fofonoff, Robert \\ Dyer, John Bevilacqua, Joshua Kaufman, Omar Roushdy, and Ian Hunter \\ Biolnstrumentation Laboratory, Massachusetts Institute of Technology. 77 Massachusetts Ave. RM. 3-147, Cambridge, \\ MA 02139-4307, USA - Tel 617258 0533, Fax 617252 1849, Email smmartel@mit.edu
}

\begin{abstract}
We propose to bring the instruments to the samples in the form of miniature wireless instrumented robots called the NanoWalkers. With the NanoWalker robot approach, instrumentations and throughput requirements can be adjusted extremely fast by simply adding, replacing, or removing robots at will. It is the aim of this project to develop a powerful and flexible environment that we believe may revolutionize the way drug, biological, material discovery, and characterization will be performed in the future.
\end{abstract}

\section{INTRODUCTION}

To give a better feel for this technology, think about an army of several hundreds or even thousands of robots called the NanoWalkers, each capable to fit in the palm of your hand and working fully-autonomously at the sub-atomic scale while executing commands received from a central computer through a fast infrared wireless communication scheme.

Based on our actual design, soon these robots will be fully assembled and will walk at a rate of at least 4,000 steps per second. Every second, each robot will execute 48 million instructions, perform 200,000 accurate measurements at the atomic scale while transmitting or receiving 4 million bits of information.

Our first prototypes demonstrated a maximum displacement rate of $200 \mathrm{~mm} / \mathrm{s}$ with $4000 \times 50$ micrometers steps/s while the minimum step size achieved so far is on the order of 30 nanometers. On the other hand, its integrated instrument will be capable of displacement well below the dimension of a single atom.

Fundamentally, the NanoWalker can be described as a highly sophisticated wireless platform capable of carrying various manipulators/actuators and instruments suited for complex molecular and atomic operations such as Scanning Tunneling Microscopy (STM), nano-manipulation, and three-dimensional micro-fabrication, just to name a few. A 4 Million bits per second (Mb/s) half-duplex infrared (IR) protocol especially adapted to distributed robotics for fully autonomous or semi-autonomous operations performed at the molecular and atomic level is under development. The NanoWalkers will also be guided through a novel positioning and navigation system capable of atomic resolution within a miniature plant that will be developed and adapted for the NanoWalker's diverse tasks.

\section{BACKGROUND}

As systems become smaller, robots [1] that are capable of inspecting, measuring various parameters, manipulating and repairing these systems or even building new systems including next generation robots at the molecular and atomic scales not only in normal but also in contaminated and/or restricted environments or even in future microfactories, will prove to be highly desirable. Using wireless and highly sophisticated miniature robots as the basic building blocks for future instruments and miniature factories [2] has several advantages for many applications. This new approach is highly modular. Such modularity allows easy scaling of the systems by adding or removing robots as required for the applications. As such, systems built with small robots become highly adaptable to new requirements and can be changed with minimum risk and delay.

Traditional systems become extremely difficult to design and to develop when the number of parallel operations required within a relatively small area increases substantially. This is due in great part to congestion between mechanical parts. Using the new approach, no extra design and development effort are needed to avoid congestion since several robots performing the same or different tasks can be added without "re-thinking" the whole system.

Economically, since "intelligence" and wireless communication must be integrated in each moving part (each robot) of such highly homogeneous system, the initial manufacturing cost could be very high. With similar robots used throughout many systems, manufacturing costs would typically decrease substantially with a higher number of units produced. Furthermore, lower development risk, extremely short development time, high flexibility, and adaptability are just a few characteristics that would likely become most critical and make this approach more economically viable in many future applications.

Since the new paradigm is to bring the instruments to the samples instead of bringing the samples to the instruments, one can envision many new applications such as thousands of biological or drug samples being inspected by a group of small robots each equipped with different instruments and all making a set of different measurements on these samples autonomously and in parallel. In manufacturing, one can also envision a group of small robots, each equipped with a set of specialized tools capable of operating at the sub-nanometer scale, as well as 
collaborating and moving through the floor of a miniature plant with nanometer accuracy. These fully autonomous robots with the capability of performing tasks at the atomic level would work together to build and assemble even smaller systems.

Such a proposed robot is called the NanoWalker [3][4][5]. The NanoWalker (Fig. 1) is a Micro-ElectroMechanical System (MEMS) of approximately 32 millimeters $(\mathrm{mm})$ in diameter. Piezo-ceramic tubes are used to implement the actuators (legs) required for motion and instrumentation. Unlike many other miniature mobile robots, the tasks performed by the NanoWalker are highly sophisticated and may include tasks at the cell and DNA levels down to the molecular and the atomic scales. As such, it requires a level of complexity [6] and microassembly [7] of the embedded electronics that far exceeds the level encountered in recent miniature robots.

Dimension: $32 \mathrm{~mm} \times 32 \mathrm{~mm}$ (instrumented version)

Infrared Emitters for $\mathrm{x}-\mathrm{y}$ and angular positioning

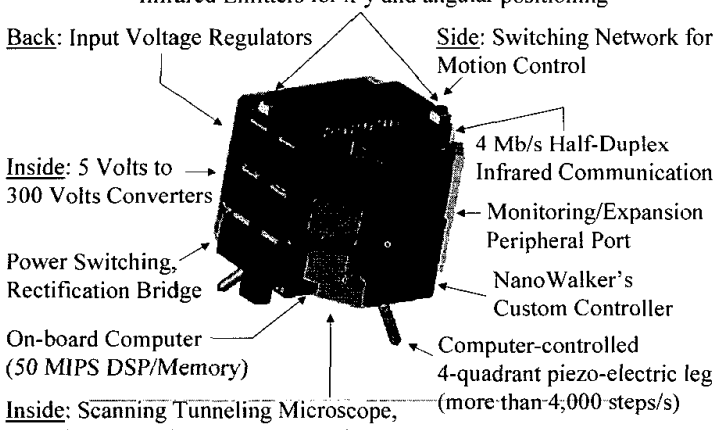

Scanning Control and Instrumentation (200,000 16-bit measurements/s)

Fig. 1. NanoWalker Robot (from [5])

Presently, several tethered prototypes have been developed and tested. New walking algorithms have been developed and evaluated through an extensive experimentation phase. Preliminary mathematical models have also been developed. The electronic system and a large percentage of the software for the final version have been developed and tested successfully including the infrared communication interfaces. The miniaturization of the electronics and the positioning system are also completed. Both the STM and 3-D fabrication facilities have been built and tested but they have not yet been integrated on the NanoWalker.

\section{LOCOMOTION}

The NanoWalker is based on a piezoelectric locomotion mechanism and architecture. [8][9][10] adapted from [11]. This type of locomotion not only provides fast displacement through several thousand steps executed per second but an extremely high resolution in displacement and positioning with step sizes in the nanometer range (hence the name "NanoWalker"). The robot is based on a three-legged architecture (Fig. 2) formed as a triangle with the apex pointing upward. Each leg is a four-quadrant piezo-ceramic tube actuator for which several parameters such as the rate and angle of deflection are controlled by sophisticated algorithms executed through complex onboard electronics.

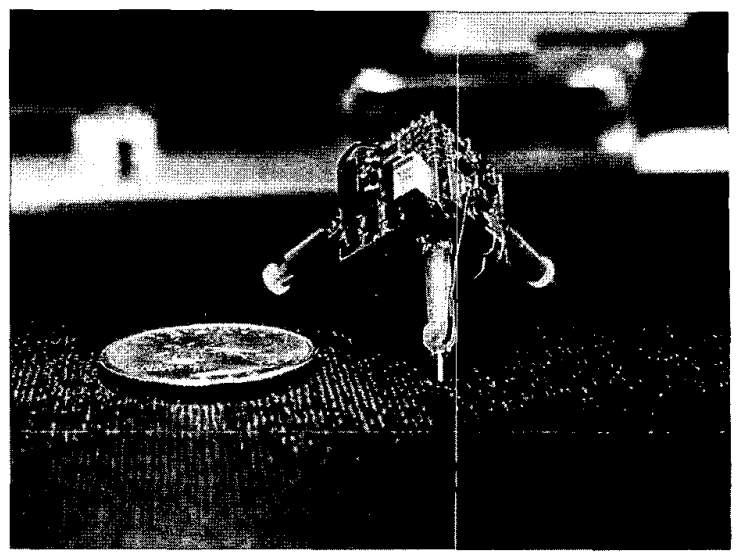

Fig. 2. Three-Legged Architecture on a Previous Wireless Prototype

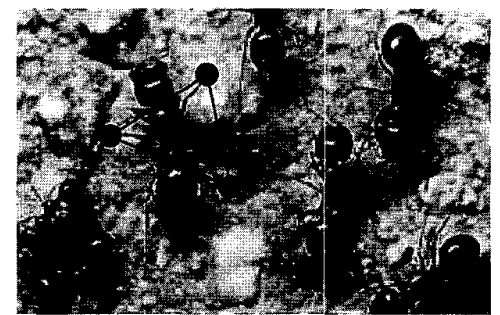

Fig. 3. Alternating Triangle of Support in Ants

The three-legged architecture provides the minimum implementation for static stability of the structure and minimizes the amount of electronics and power required for motion control. Unlike terrestrial insects that make use of alternating triangles of support (Fig. 3) during walking and running, the NanoWalker relies on a single triangle of support during motion. Hence, as soon as a leg is lifted, the structure becomes unstable. Therefore, each step must be executed extremely fast with tightly controlled synchronization between the legs. Furthermore, the required accuracy in step sizes makes the robot motion very sensitive to various parameters such as material (coefficient of friction), weight, inertia, and various static and kinetic forces that must be considered in the development of new walking algorithms, as well as mechanical and electronic systems that can adapt to various conditions.

At running speeds, worker ants for instance continue to use alternating triangles of support [12] but there are periods when all the legs are off the ground at the same time. The worker ants appear to "bounce" from one triangle of support to the next. Similarly, at running speeds previous prototypes of the NanoWalker have shown step sizes at 
high as 25 to 50 micrometers, larger than the leg's maximum deflection of a few micrometers indicating that "bouncing" was taking place [8][10]. Under these conditions, the stability of insects [13] and the NanoWalker provided by the triangle of support is greatly reduced; and dynamic stability as well as the need for corrective actions by sophisticated onboard electronics and computer becomes increasingly critical as the displacement speed increases.

\section{BASIC PRINCIPLE BEHIND A SINGLE STEP}

Several walking strategies have been developed and the theory behind each of these strategies is quite complex and beyond the scope of this paper. Nonetheless, the general principle considering a single leg at a time will be briefly described.
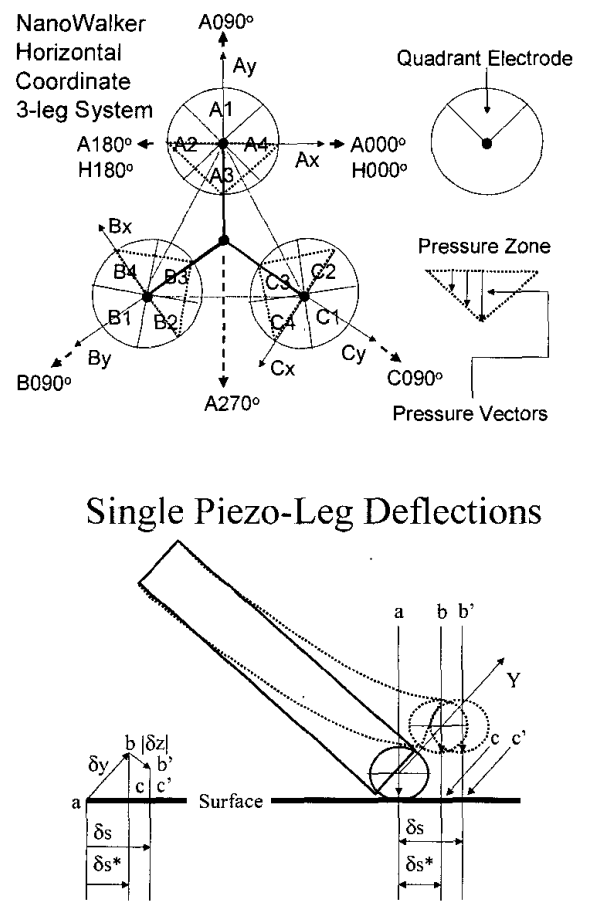

Fig. 4. Piezo-Ceramic Legs (from [9]) (not to scale)

A leg is made of a piezo-ceramic tube. In our particular case, the piezo-ceramic material used is lead zirconate titanate chosen particularly for its fine displacement. The dimensions of a piezo-ceramic element change when stressed by a voltage. The electrical stress is delivered to the legs through the quadrant electrodes A1..4, B1..4, C1..4 for legs A, B, and C, see Fig. 4.

The stress in the form of voltage results in a strain or mechanical distortion of the piezoelectric legs, which is proportional to the polarization. The motion of the NanoWalker is performed mainly through deflection of the piezo-ceramic tubes in the bending mode. For instance, bending leg A toward the direction A090 in Fig. 4 would require a voltage across electrodes $\mathrm{A} 1$ and $\mathrm{A} 3$. By applying voltages of same polarity simultaneously to all four external electrodes, it is also possible to contract or extend the leg. This is referred to as the stretching mode. For longer legs, the maximum amplitude of deflection is larger in the bending mode than in the stretching mode. As the legs become shorter, the locomotion cannot be based primarily on the bending amplitude but rather on the stretching amplitudes. As such, the control algorithm behind the locomotion must adapt adequately.

The NanoWalker will use a walking method determined by several factors such as the geometry of the legs, its total weight, the capabilities of the onboard electronics, and more importantly, the type of walking surface. Basically, the NanoWalker will use at various speeds, orientations, and synchronizations between the legs, three basic types of motions for each leg at any given time: lifting, pushing, or a combination of both. Examples of typical displacement rates obtained experimentally at relatively low frequencies (steps/s) with a substantial mass (182 grams) that may be caused by the onboard electronic systems are shown in Fig. 5.

Knowing that the piezo-ceramic legs are capable of extremely fast angular acceleration, from Newton's second law, it can be estimated that this will generate a corresponding force or torque which will depend on the total moment of inertia of the leg being the sum of the tube and the foot inertia. Therefore, the length of the legs and the mass of the feet are important factors to consider. At an angle of $45^{\circ}$ from the surface, each leg has a corresponding vertical (lifting) and horizontal force components proportional to the angular acceleration.

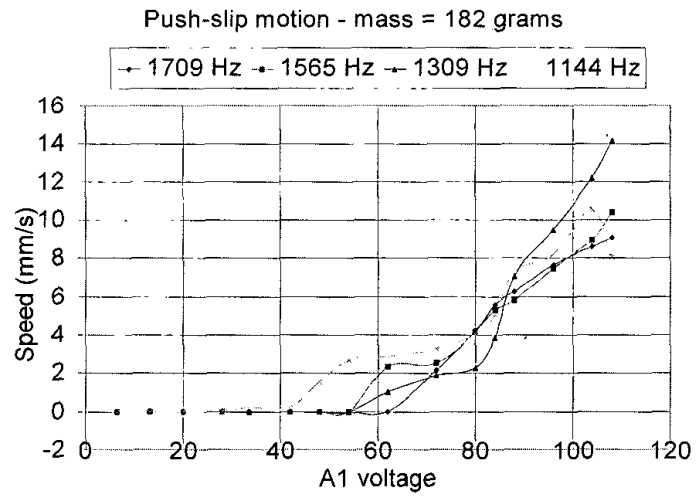

Fig. 5. Example of Results in Locomotion (from [10])

Although the onboard electronics can generate electrical stress at a very fast rate, the angular acceleration of the legs can be increased substantially by using the friction between the feet and the walking surface. Initially, if there is a corresponding static coefficient of friction between the feet and the walking surface, an initial bending force will build up to the maximum reciprocal static force of friction proportional to the normal force which increases with an increase of the total mass of the robot. When the legs enter the kinematic state (i.e. begin motion), this initial bending force creates an immediate additional acceleration leading to additional torque and then a "boost" in vertical lifting force. For a short time, the kinetic force of friction, which is always less than the maximum static force of 
friction, will act against the leg motion and reduce the angular acceleration. Therefore, the feet are designed with a geometry suited to minimizing the impact of the kinetic force of friction.

\section{WIRELESS ROBOTS}

The optimal goal is to implement a wireless robot since past experiments have shown that even the tiny wires presently used on the tethered prototype version of the NanoWalker significantly affect the accuracy in motion. Hence, although a wireless version is highly desirable to avoid tangled wires when several robots are working within the same workspace, a wireless version is also essential to maintain maximum accuracy in motion.

The implementation of the embedded electronics in such a wireless system alone is a complex process [6]. The design by itself requires various areas of expertise in analog, digital, communication, instrumentation, and embedded processing. More specifically, the design involves the development of advanced infrared communication interfaces, custom devices, embedded processing blocks based on digital signal processing, power circuits, in-system programming and management, and various analog-based functions, just to name a few.

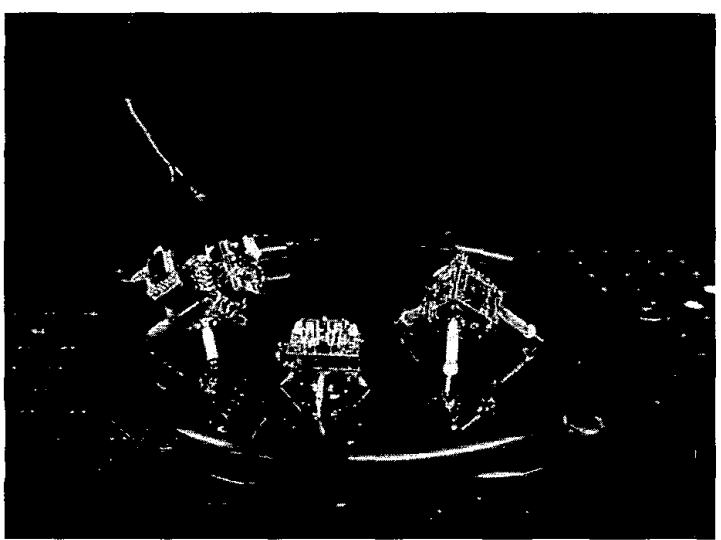

Fig. 6. Three Simpler Prototypes

Because of the extremely small form factor of the final electronic assembly, the implementation process requires the development of larger prototypes that can be probed by various instruments such as logic/states analyzers, additional electronic boards especially designed for insystem programming and monitoring, and several simpler prototypes [14] of the NanoWalkers (Fig. 6) to test and validate new concepts and ideas. As such, substantial efforts are targeted at hardware/software co-verification, validation, and simplification prior to implementation at the final miniaturization scale.

The final micro-assembly is also a critical phase and is restricted by several technological constraints. The electronic system, although extremely sophisticated and powerful, relies on a large number of electronic components (several hundreds) that must be integrated using very advanced modern micro-assembly and packaging techniques [6]. As such, stud bumping with flipchip assembly [7] is the preferred method at the present time.

The power source and power conversion and other critical factors such as coupling noises and thermal dissipation currently constrain the level of miniaturization. To miniaturize the robot beyond the level achievable with more traditional approaches, several new techniques have been developed. One example is the implementation of a very thin (approximately $1 \mathrm{~mm}$ thickness) heat dissipation "skin" which is significantly smaller than a standard heatsink. It dissipates heat by evaporating distilled water stored in a few layers of flexible patterned wiping fabric designed for maximum water absorption and encapsulated between two layers of thermally conductive elastomer (e.g. Thermagon T-pli 200 series). The "skin" is re-hydrated by allowing each robot to "drink" when necessary.

\section{INSTRUMENT}

The present prototype system has all the electronics to control the STM tip and to record STM-based images. The methods of recording will be similar to the methods used in conventional scanning tunneling microscopes [15]. The STM is an extremely powerful tool since it can resolve local electronic structure at an atomic scale on every kind of conducting solid surface, allowing its local atomic structure to be revealed. The measurement surface is "grounded" through the reference leg (one of the legs of the robot as explained later) and the robot generates a bias voltage through the STM tip. The embedded processor will move the tip towards the surface until a tunneling current is recorded. Such a current is generated when the distance between the tip and the surface is so small that a few electrons escape due to the bias voltage by transiting the small gab between the tip and the surface. The robot then performs a scan by controlling the deflection of the STM piezo-ceramic tube. Although several scanning methods are possible, our first method will maintain the tunneling current constant by moving the tip vertically and maintaining a constant distance between the tip and the electron cloud spinning around the nucleus. This small current is amplified by a current amplifier, which is integrated in the robot. The amplified current is then converted in digital form to make it accessible to the computer onboard the NanoWalker. Based on the converted values, which indicate the distances between the tip and the surface, the NanoWalker maintains a constant distance when the region of interest is being scanned. The position of the tip will also be transmitted back through the wireless infrared communication link to the central computer to create the image. We have already built STM prototypes and recorded satisfactory images of atoms.

\section{COMMUNICATION, POSITION AND NAVIGATION}

The position and navigation infrastructure [16] for the NanoWalker is based on two positioning systems: the mandatory Global Scale Positioning System (GSPS) and the optional Atomic Scale Positioning System (ASPS) integrated into one of possibly many communication cells 
of 0.5 meter in diameter each. One communication cell is depicted in Fig. 7.

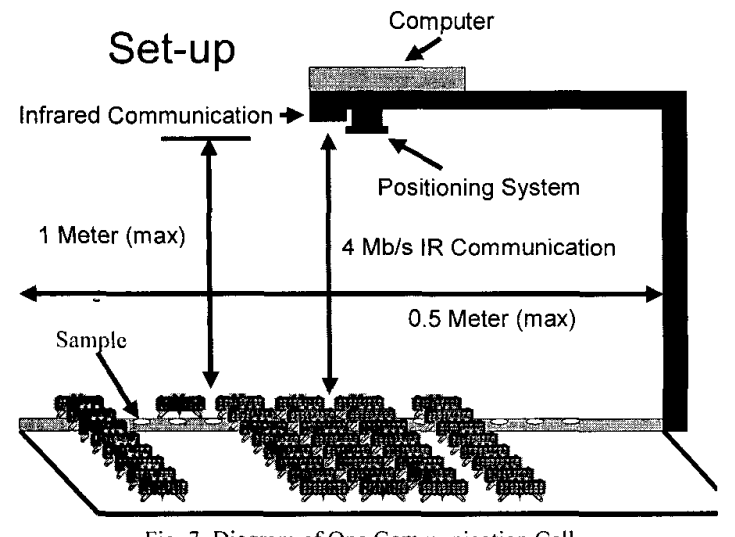

Fig. 7. Diagram of One Communication Cell

The goal of the GSPS is to provide accurate positional information within a workspace having a maximum diameter of 0.5 meter. This diameter corresponds to the size of the infrared (IR) communication cell at the maximum transmission speed of $4 \mathrm{Mb} / \mathrm{s}$. Notice that the range can be extended through several cells (like in the case of cellular phone technology). The GSPS is based on Position Sensing Detection (PSD) technique. The resolution of the GSPS as depicted in Fig. 8 also matches the maximum range of the ASPS which is determined by the maximum deflection of the embedded STM tip as mentioned later. Since such resolution is only required when the robot reaches its final destination, it is anticipated that other parameters such as the covered area and/or the rate of positioning feedback may vary dynamically as the robot walking speed gradually decreases when moving toward its final destination. Decreasing the speed will allow better resolution through longer integration time. Angular information will also be provided with a second intermittent IR source mounted on each robot.

With the GSPS, the position of each robot is presently detected using the 870 -nanometer $(\mathrm{nm})$ wavelength IR transmissions originating from the robot. If the last transmission from the robot corresponds to a request for Global Scale Position (GSP) according to the NanoWalker communication protocol, the GSPS will transmit back its position through the same IR communication link. The robot will then take the position as feedback to correct its trajectory in order to reach its final assigned position. The GSP system is also used as a control tower to inform every robot of the position of other robots such that no collision occurs during travel.

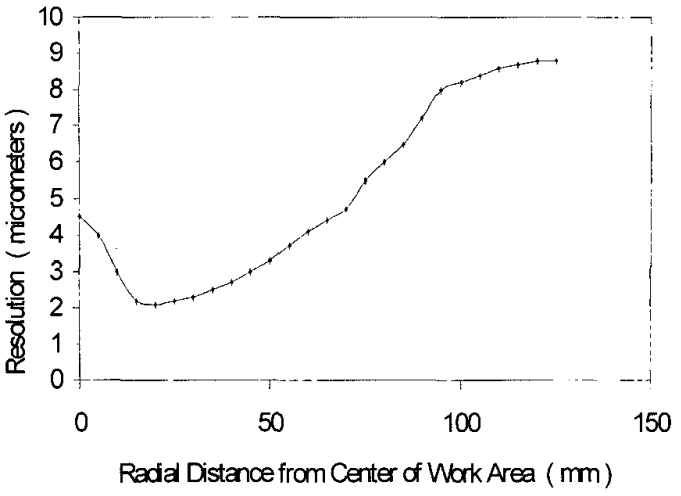

Fig. 8. Resolution of the PSD-based System (from [16])

The ASPS will be essential for work down to the atomic scale and for grouped work (i.e. work being done by more than one robot at the same location but equipped with different tools) again at the atomic or molecular scale. The ASPS will allow another NanoWalker to position itself at exactly the same location and continue the work at the atomic scale of a previous NanoWalker that has left its working position. The maximum range of the ASPS will correspond to the maximum deflection of the STM tip since once at the GSP, the robot will first scan the region using STM technique to localize a reference point previously made by the first NanoWalker involved in the work and then by "counting" the number of atoms to determine the final position.

\section{POWER FLOOR}

The power floor [17] is a special flat walking surface with insulated patterns carrying the power to various working cells distributed throughout the surface. With this approach, the NanoWalkers have continuous autonomy by delivering power through the robot's feet.

As depicted in Fig. 9, the power floor is divided into several power bands of alternating polarities. Narrow insulation bands prevent electrical short between the power bands. As the robot walks on the floor, the dimensions of the bands are set to allow one leg to be in contact with a positive band while another leg is in contact with a negative band such that the supply current can flow through the embedded electronic system of the robot. A special switching circuit embedded in each robot allows each leg to support either of the two polarities at any given time. Since the electrical current used to power the robots would prevent the measurement of tiny tunneling current during STM-based operations, neutral zones are defined on the walking surface. Each neutral zone has a conductive surface insulated from the power bands. The samples to be analyzed and/or manipulated are placed on the neutral zones. When in working position, the NanoWalker would then have one power leg on a positive power band, another power leg on a negative power band, and the last leg (the reference leg) on a neutral zone. There are several other technical issues that have been addressed in the fabrication of the power floor. The width of the power bands for 
instance is dependent on the dimensions of the robot. The constraints imposed by the neutral zones call for assistance from the infrared positioning system. Special feet have also been designed to transit the insulation bands with minimum change in the motion behavior and without causing electrical short during the transitions at various angles. They also provide low impedance through sufficient contact area to provide without interruption the required power to the robot's embedded electronic system. The normal force distributed to each leg caused by the relatively high mass of the robot significantly reduces the electrical resistance with the power floor.
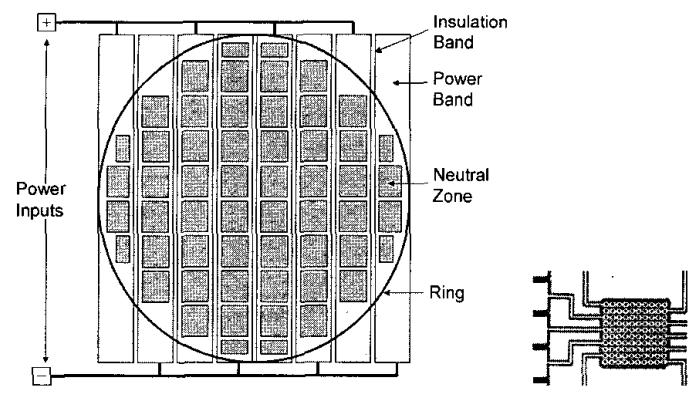

Fig. 9. Basic Diagram of the Power Floor

Neutral zones can simply be a flat surface but also a more specialized surface with embedded structures such as the one shown in the right corner of Fig. 9., where microwells have been implemented to retain small amount (micro-liters) of liquid. In this example, designed and built in our laboratory, electrical measurements can also be done through the special conductive rings surrounding each well. The robot can also perform additional tests as well as pumping and mixing different concentrations. Many other structures are possible depending on the applications.

\section{SUMMARY}

A new environment where conventional instruments are replaced by miniature three-legged robots has been briefly described. The architecture of the NanoWalker instrumented robot has also been presented with a basic description of its locomotion system based on piezoceramic actuators. Prototypes have shown step sizes as large as 50 micrometers and as small as 30 nanometers when operating at 4000 steps per second. The robot is initially designed to have a scanning tunneling microscope tip that will allow it to operate at the sub-atomic scale.

\section{ACKNOWLEDGMENT}

We gratefully acknowledge support from the Seaver Institute.

\section{REFERENCES}

[1] S. Fatikow, B. Magnussen, and U. Rembold, "A piezoelectric mobile robot for handling of micro-objects," Proc. of the Int. Symp. on Microsystems, Intelligent Materials and Robots, Sendai, 1995.
[2] J. Breguet, C. Schmitt, and R. Clavel, "Micro/nanofactory: Concept and state of the art," Proceedings of SPIE Microrobotics and Microassembly, Vol. 4194, Boston, USA, pp. 1-12, Nov. 2000 .

[3] S. Martel, P. Madden, L. Sosnowski, I. Hunter, and S. Lafontaine, "NanoWalker: a fully autonomous highly integrated miniature robot for nano-scale measurements," Proceedings of the European Optical Society (EOS) and SPIE International Symposium on Envirosense, Microsystems Metrology and Inspection, Vol. 3825, Munich, Germany, 12 pages, June 14-18, 1999.

[4] S. Martel, J. Bevilacqua, R. Dyer, T. Fofonoff, W. Garcia de Quevedo, C. Helm, M. Sherwood, and I. Hunter, "Development of a miniature three-legged bio-instrumented autonomous robot," IEEE-EMBS Asia-Pacific Conference on Biomedical Engineering, Hangzhou, China, pp. 513-514, Sept. 26-28, 2000.

[5] S. Martel, O. Roushdy, and I. Hunter, "Miniature instrumented robots for mass-scale synthesis and characterization," 1 'st Anmual International IEEE-EMBS Special Topic Conference on Microtechnologies in Medicine \& Biology, Lyon, France, pp. 160164, Oct. 12-14, 2000.

[6] S. Martel, K. Doyle, G. Martinez, I. Hunter, and S. Lafontaine, "Integrating a complex electronic system in a small-scale autonomous instrumented robot: the NanoWalker project," Proceedings. of SPIE, Microrobotics and Microassembly, Vol. 3834, Boston, USA, pp. 63-74, Sept. 1999.

[7] S. Martel S., G. Riley, M. Merchant, I. Hunter, and S. Lafontaine, "Flip chip electronic system assembly process and issues for the NanoWalker: a small wireless autonomous instrumented robot," Proceedings of SPIE, Microrobotics and Microassembly, Vol. 3834, Boston, USA, pp. 55-62, Sept. 1999.

[8] S. Martel, M. Szabelski, A. Leija, P. Madden, S. Lafontaine, and I. Hunter, "Initial results of a new type of locomotion for a wireless instrumented robot capable of subatomic movements," Proceedings of the Biomedical Engineering Society and the 21st Annual International Conference of the IEEE Engineering in Medicine and Biology Society, Atlanta, GA., Oct. 13-16, 1999.

[9] S. Martel, A. Saraswat, and I. Hunter, "Fundamentals of piezoceramic actuation for micrometer and sub-micrometer motions for the NanoWalker robot," Proceeding of SPIE, Microrobotics and Microassembly, Vol. 4194, Boston, USA, pp. 82-93, Nov. 2000.

[10] S. Martel, A. Saraswat, A. Michel, and I. Hunter, "Preliminary evaluation and experimentation of the push-slip method for achieving micrometer and sub-micrometer step sizes with a miniature piezo-actuated three-legged robot operating under high normal forces," Proceeding of SPIE, Microrobotics and Microassembly, Vol. 4194, Boston, USA, pp. 141-148, Nov. 2000.

[11] R. Munassypov, B. Grossmann, B. Magnussen, and S. Fatikow, "Development and control of piezoelectric actuators for the mobile micromanipulation system," Proceeding Actuator, Bremen, pp. $213-216,1996$

[12] C.P.E. Zollikofer, "Stepping pattern in ants," Journal of Experimental Biology, Vol. 192, pp. 119-127, 1994

[13] L.H. Ting, R. Blickham, and R.J. Full, "Dynamic and static stability in hexapedal runners," Journal of Experimental. Biology, Vol. 197, pp. 251-269, 1994.

[14] S. Martel, J. Kaufman, C. Helm, T. Fofonoff, J. Bevilacka, R. Dyer, J. Levine, J. Au, and I. Hunter, "NanoRunner: a very small wireless robot with three piezo-actuated legs suited for design experimentations and validations through pre-programmed behaviors," Proceeding of SPIE, Microrobotics and Microassembly, Vol, 4194, Boston, USA, pp. 149-156, Nov. 2000.

[15] C. Chen, "Introduction to scanning tunneling microscopy," Oxford University Press, New York, 1993.

[16] S. Martel, O. Roushdy, M. Sherwood, and 1. Hunter, "Highresolution optical positioning system for miniature robot," Proceeding of SPIE, Microrobotics and Microassembly, Vol. 4194, Boston, USA, pp. 121-128, Nov. 2000.

[17] S. Martel, W. Garcia de Quevedo, and I. Hunter, "Techniques for continuous power delivery to a group of 15 -Watt +3.3 to \pm 150 VDC miniature wireless instrumented and fast stepping robots through several thousand intermittent contacts per second between the robots' legs and the walking surface," Proceeding of SPIE, Microrobotics and Microassembly, Vol. 4194, Boston, USA, pp. 168-177, Nov. 2000. 